\title{
Glucose Intolerance, CTCAE
}

National Cancer Institute

\section{Source}

National Cancer Institute. Glucose Intolerance, CTCAE. NCI Thesaurus. Code C143519.

A disorder characterized by an inability to properly metabolize glucose. 